\title{
A Unified QoS-Inspired Load Optimization Framework for Multiple Access Points Based Wireless LANs
}

\author{
Eng Hwee Ong, Jamil Y. Khan \\ School of Electrical Engineering and Computer Science \\ University of Newcastle, Australia, NSW 2308 \\ Email: enghwee.ong@studentmail.newcastle.edu.au, jamil.khan@ newcastle.edu.au
}

\begin{abstract}
The extensive deployment of 802.11 WLAN has positioned itself as the de-facto wireless access network for the 'last mile' connections. The potential for WLAN to deliver multimedia contents such as VoIP, video conferencing, streaming and data services will become a reality with the advent of $802.11 \mathrm{n}$ standard, promising data rate of up to $600 \mathrm{Mbps}$. Although these QoS demanding multimedia applications can be supported by the 802.11e standard, it offers limited QoS guarantee. Further, its possible adoption by the industry remains unclear due to high cost of hardware replacements. In this paper, we give a solution to provision QoS support for multimedia traffic in commercially deployed $802.11 \mathrm{a} / \mathrm{b} / \mathrm{g}$ WLAN operating with fundamental distributed coordination function (DCF) access mechanism in a unifying QoS-inspired load optimization framework. We further postulate the notion of $\mathrm{QoS}$ balance as load criterion in heterogeneous WLANs where data rates and channel conditions are radically different. In practice, link adaptation employed to combat diverse channel conditions could lead to multirate operation and consequently the long-term throughput of stations are penalized by the lowest data rate peer. We argue that such rate anomaly can be mitigated by maintaining a QoS-balanced system. Simulations show that our solution provides service QoS guarantee for both real-time and non real-time traffic as well as achieves both throughput and QoS fairness under dynamic network conditions in a self-adjusting manner.
\end{abstract}

\section{INTRODUCTION}

The 802.11 WLANs are already pervasive in many diverse environment such as enterprises, universities, hotels and public hotspots due to its low cost deployment. The forthcoming 802.11 n standard offering data rate of up to $600 \mathrm{Mbps}$ will accentuate its benefits for high-speed ubiquitous broadband wireless access. However, delivering QoS demanding multimedia applications such as VoIP and video over IP in presence of data services over WLAN are very challenging. First, WLAN requires service differentiation which is lacking in DCF channel access mechanism that accounts for a majority of the currently deployed WLANs. Although the emerging 802.11e standard supports service differentiation, it cannot guarantee strict QoS required by real-time services under heavy load without an appropriate admission control or load balancing scheme [1],[2]. Moreover, its adoption by the industry is questionable due to significant capital expenditure involved in replacing existing $802.11 \mathrm{a} / \mathrm{b} / \mathrm{g}$ WLAN hardwares for additional QoS support. A possible alternative [3] is implementing a dual queue strategy at the access point device driver level requiring only a software upgrade to provide service prioritization on existing DCF-based access points.

Second, WLAN requires knowledge about the radio environment in order to improve performance and provide guarantee to multiple QoS requirements of multimedia applications. For example, measurements such as load information of neighboring access points are important for load balancing function. Moreover, average media access delay of access points could be used for selecting an access point in a dynamic and adaptive manner that best meets a user service QoS requirements while preventing unnecessary handovers [4]. More importantly, channel impairments are apparent in hotspot deployments and indoor environments where frequent non-line-of-sight (NLOS) transmissions caused by structures and obstacles should be considered together with network load, achievable network QoS performance and desired service QoS when running load balancing algorithm. The recently approved $802.11 \mathrm{k}$ standard provides such radio resource measurements which are exploited in this work to facilitate an intelligent self-adjusting WLAN network.

Third, rate anomaly [5] occurs when contending nodes in the same BSS with similar channel conditions transmit packets of same size but with different data rates. Under such conditions, high data rate nodes would yield same throughput as the lowest data rate node according to [6]. The root cause of rate anomaly stems down to throughput fairness of DCF that dictates equal probability of channel access. In other words, slower rate nodes disadvantage higher rate nodes by occupying more channel time to acquire approximately the same transmission opportunities. For example, the aggregate throughput of multirate transmissions by two stations using $11 \mathrm{Mbps}$ and $1 M b p s$ is much lesser than the average of the total throughput that could be achieved when both stations transmit using either $11 M b p s$ or $1 M b p s$. This creates severe capacity outages when the arriving traffic exceeds the downgraded system capacity. Hence, an important implication of rate anomaly is that it dilutes the benefits derived from any link adaptation techniques used to exploit the tradeoff between date rate and bit error rate under varying channel conditions. This impact would be magnified under heavy load situations and dissimilar channel conditions which will trigger multirate operation.

In this paper, we propose a holistic approach to provision 
QoS in presence of the above multifaceted challenges by redistribution of load across a multiple access points based WLANs opportunistically, where access points are physically co-located. The basic idea is to exploit diversities of such multiple access points based WLANs. This promotes a QoS-balanced system which inherently provides service QoS guarantee for both real-time and non real-time traffic and maximizes global system capacity in a self-adjusting manner. To the best of our knowledge, there is no prior art on load balancing scheme that offers service QoS guarantee and optimizes system capacity from a unified perspective, under network heterogeneity such as data rates and dissimilar channel conditions. The remainder of the paper is organized as follows. Section II discusses related work. Section III describes our proposed QoS-inspired load optimization framework. Section IV illustrates the simulation model. Section V presents the simulation results and Section VI concludes the paper.

\section{RELATED WORK}

The choice of appropriate load metrics is pivotal in any load balancing schemes as it serves to estimate the available network capacity. For example, packet loss rate and retransmissions suggested by Bianchi et al. [7] and media access delay proposed by Ong et al. [8] are reflective of channel conditions, whereas channel utilization offered by Garg et al. [9] gives the best representation of the effective network load.

Accordingly, channel utilization which measures the fraction of channel occupation time per observation interval has been widely used as load metric for both load balancing and admission control algorithms due to its simplicity and high accuracy in estimating effective network load. Zhai et al. [1] employed channel busyness ratio as load metric for their admission control and rate control scheme. However, the authors did not consider load balancing feature in their work. On the other hand, Daher et al. [10] considered load balancing and incorporated admission control within a centralized architecture using medium busy time as load metric. However, this approach requires additional central load controller which increases network signaling overheads, creates bottleneck and prone to single point of failure.

Velayos et al. [11] proposed a decentralized load balancing scheme using throughput per access point as their load metric. However, throughput according to [9] is not a suitable load metric as they are highly influenced by data rate of stations running different applications and variable transmission data rate due to dynamic channel conditions affecting link quality. Collectively, these previous works consider load balancing and admission control in the context of ideal similar channel conditions where a single load metric suffice. However, we argue that using channel utilization as a single load metric under dissimilar channel conditions has catastrophic effect.

To this end, we note that numerous solutions have been proposed to address rate anomaly problem of DCF prevalent in multirate WLAN. Most notable works approach this problem from temporal fairness [6] or proportional fairness instead of throughput fairness. However, we take a completely different approach by treating rate anomaly from a QoS perspective. We advocate that by identifying users with degrading service QoS and maintaining a QoS-balanced system, capacity outage time inflicted by any link adaptation procedures can be substantially reduced. This will act to maximize the available system capacity globally (cf. $\S \mathrm{V}-\mathrm{B}$ ).

Our contributions differ from related works in four significant ways: (i) our proposed QoS-inspired load optimization framework gives a unifying treatment in QoS provision for multimedia traffic over the widely deployed DCF-based WLANs; (ii) we exploit quadruple load metric consisting of packet delay, packet loss rate, missed acknowledgment and channel utilization for adaptation to dynamic network conditions which include traffic and channel variations; (iii) we introduce the notion of $Q o S$ balance, as load criterion in heterogeneous WLANs, which has intrinsic properties of providing service QoS guarantee while maximizing global system capacity; and (iv) we argue that rate anomaly arising from link adaptation can be mitigated through QoS balancing by minimizing the possible capacity outage time.

\section{PRoposed QoS-INSPiRed LoAd Optimization FRAMEWORK}

The fundamental of our proposed QoS-inspired load optimization framework is network-assisted discovery [8], such that source access point broadcasts measurement report of neighboring access points together with its own. This concept is compatible with the IEEE 802.21 media independent handover services where access to information about different networks within a geographical area can help in the handover decision making process and allow optimal power utilization. By listening to broadcasts, stations would acquire state information of packet delay (PD), packet loss rate (PLR) and channel utilization (CU) as inputs for network selection, handover control, admission control and load control to optimize load distribution across multiple access points based WLANs opportunistically in a distributed and selfadjusting manner. As illustrated in Fig. 1, our solution provides adaptation to different services with network-QoS entity and dynamic network conditions through connection-QoS entity.

The network-QoS entity consists of both service prioritization and admission control to deal with different user service profiles. Multimedia traffic can be classified into real-time and non real-time according to their delay requirements. Real-time traffic such as VoIP and video conferencing are delay-sensitive whereas non real-time traffic, also known as elastic traffic, can tolerate a relatively larger delay. Hence, it is important to introduce service prioritization in DCF-based WLAN such that real-time traffic can be handled with higher priority than non real-time traffic, in order to support differentiated QoS of multimedia traffic. For this purpose, we adopt the dual queue strategy proposed in [3] with a slight modification to introduce an additional granularity of service prioritization to support voice, video and data flows. The admission control regulates the network load by operating under the saturation threshold in order to protect QoS of existing flows. In fact, admission 


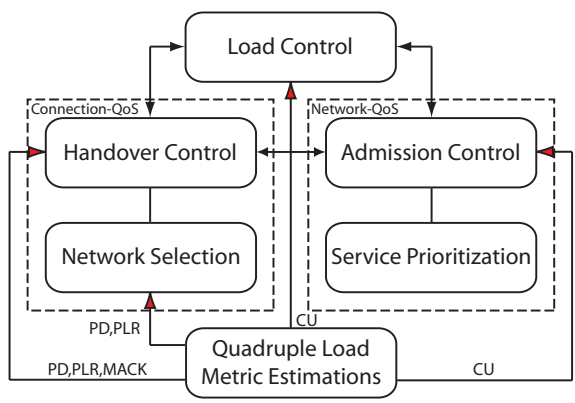

Fig. 1. Distributed QoS-inspired load optimization framework.

control and load control are often not dissociable. The main reason is that both rely on the knowledge of load metric in order to make their decision. Hence, we choose the channel utilization estimation technique suggested in [9] as load metric for both admission control and load control. Accordingly, the $\mathrm{CU}$ of each flow and the corresponding network capacity are estimated at every beacon interval as,

$$
\begin{gathered}
C U_{\text {total }}^{n}=\sum_{j \in \text { flows }} C U_{j}^{n}, \quad n=1, \ldots, N, \\
C U_{j}^{n}+C U_{\text {total }}^{n}<C U_{\text {max }},
\end{gathered}
$$

where $0 \leq C U_{\text {total }}^{n} \leq 1$ is total channel utilization of $n$th access point, $C U_{j}^{n}$ is channel utilization of $j$ th flow and $C U_{\max }$ is the saturation threshold. A new flow can be accepted without affecting QoS of existing flows if Eq. 1 is true.

On the other hand, connection-QoS entity consists of both network selection and handover control to deal with dynamic network conditions associated with channel impairments and network congestions. We utilize PD and PLR which are the critical QoS parameters for multimedia traffic as metrics for network selection [4] to reliably select the 'best' access point according to the QoS requirements of different traffic classes. The handover control incorporates fast handover [8] to support real-time services by eliminating both detection and scanning delay as information of the 'best' target access point is known.

Under the notion of QoS-balanced system, a handover will be triggered only if service QoS could not be sustained and a better quality access point exists. This design philosophy is motivated by the need to reduce unnecessary handovers. Imminent handover is detected when PLR of the access point, which is the bottleneck for infrastructure-based WLAN in presence of many two-way VoIP or video conference connections [8], exceeds $1 \%$. An additional missed acknowledgment (MACK) metric is necessary for handover control function of access point to distinguish between traffic and channel variations. The idea of MACK is similar to automatic rate fallback employed in Lucent WaveLAN-II. Specifically, consecutive MACKs signify bad channel conditions, while PD denotes serious service QoS degradation due to abrupt traffic variations. Although MACK could also occur due to collisions, we argue that probability of collisions is small when WLAN is unsaturated [1] as admission control can regulate input traffic accurately with CU. Accordingly, a station requests to perform handover when PD exceeds the required threshold. When bad channel condition is detected, the access point would nominate a 'worst' station to perform handover as,

$$
S T A_{\text {worst }}^{n}=\max _{i \in M}\left(M A C K_{i}^{n}\right) .
$$

Otherwise, the access point would nominate a 'best' station to perform handover as,

$$
\begin{gathered}
S T A_{\text {best }}^{n}=\min _{i \in M}\left(C U_{\text {ave }}-C U_{\text {total }}^{m}-C U_{i}^{n}\right), \\
\text { s.t. } C U_{\text {ave }}-C U_{\text {total }}^{m}>0, \quad n, m=1, \ldots, N, \quad m \neq n,
\end{gathered}
$$

where $C U_{\text {ave }}$ is average channel utilization of $N$ access points and the constraint prevents load distribution to an overloaded access point. Although handover is crucial for both mobility and load balancing, this work focuses on vertical handovers to redistribute load to a better quality or less loaded access point opportunistically.

\section{Simulation Model}

The simulation models are developed using OPNET $^{\mathrm{TM}}$ Modeler ${ }^{\circledR} 14.0$ with wireless module. We perform modifications to the existing DCF model to provide QoS support with service prioritization, admission control, network selection, handover control and load control mechanisms which are the focus of this study. We also enhance the physical layer model to include shadow fading and the capability to simulate non-line-of-sight (NLOS) transmissions by varying the path loss exponent. We further model the network interface parameters according to Cisco Aironet 1130AG series access point and CB21AG series wireless client reference interface specifications to improve the realism of our simulations.

\section{A. Channel Model}

In this paper, we consider an error-prone channel by including shadow fading and variable path loss exponent to capture different propagation environments which may contain obstacles to cause NLOS transmissions. Accordingly, the lognormal path loss model [12] is derived as,

$$
L_{\text {path }}(d B)=10 \log \left[\frac{(4 \pi)^{2} d^{n}}{\lambda^{2}}\right]+\mathrm{X}_{\sigma},
$$

where $\lambda$ is wavelength associated with the carrier frequency, $d$ is distance between transmitters and receivers, $n$ is path loss exponent which determines the rate of loss and $\mathrm{X}_{\sigma}$ is a zero-mean Gaussian distributed random variable with standard deviation $\sigma$ (in $\mathrm{dB}$ ). We simulate NLOS transmissions due to obstructions prevalent in hotspots and indoor environments by varying $n$ uniformly $U[3,4]$ and account for shadow fading by varying $\sigma$ uniformly $U[6,9]$. We also model background noise by factoring in receiver noise figure simulated with a normal distribution $N(5,0.1)$.

\section{B. Network Configurations}

Without loss of generality, we use the wireline-to-wireless network topology in order to focus on delay within each BSS. Real-time traffic such as VoIP and video requires oneway end-to-end delay of less than $150 \mathrm{~ms}$ according to ITUT G.114 recommendation. We assume codec delay of $40 \mathrm{~ms}$, 


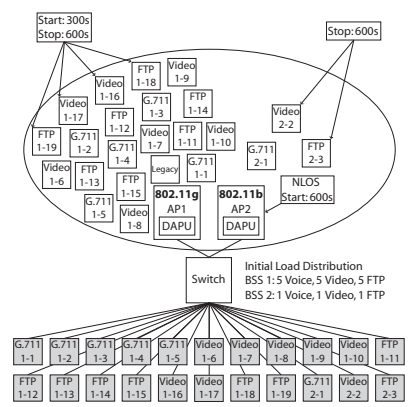

(a) Scenario 1 .

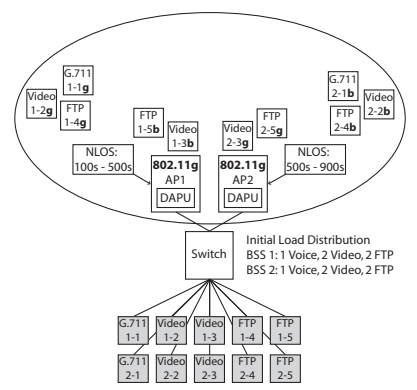

(b) Scenario 2 .
Fig. 2. Simulation models.

TABLE I

TRAFFIC GENERATION PARAMETERS.

\begin{tabular}{cccc}
\hline \hline $\begin{array}{c}\text { Traffic } \\
\text { Type }\end{array}$ & $\begin{array}{c}\text { Packet Size } \\
\text { (Bytes) }\end{array}$ & $\begin{array}{c}\text { Inter-arrival } \\
(\mathrm{ms})\end{array}$ & $\begin{array}{c}\text { Avg. Data Rate } \\
\text { (kbps) }\end{array}$ \\
\hline Voice-CBR (G.711) & 80 & 10 & 64 \\
Video-CBR & 1000 & 125 & 64 \\
Data-FTP (UL) & 450 & 120 & 30 \\
Data-FTP (DL) & 1500 & 40 & 300 \\
\hline
\end{tabular}

packetization delay of $20 \mathrm{~ms}$ at both sender and receiver and backbone network delay of $30 \mathrm{~ms}$. Therefore, the wireless network delay in both uplink and downlink should be less than $60 \mathrm{~ms}$ in order to meet the one-way end-to-end delay requirement of real-time packets.

For scenario 1, we simulate a hotspot with one $802.11 \mathrm{~b}$ and one $802.11 \mathrm{~g}$ access point, operating with maximum data rate of $11 \mathrm{Mbps}$ and $54 \mathrm{Mbps}$ respectively as depicted in Fig. 2 (a). We assume that at least one legacy station is associated with the $802.11 \mathrm{~g}$ access point since it is unlikely to operate in $802.11 \mathrm{~g}$ only mode given the vast $802.11 \mathrm{~b}$ deployments. However, the legacy station does not transmit any traffic so that all the system resources are available for $802.11 \mathrm{~g}$ stations. We initially introduce an unbalanced load of five G.711, five video, five FTP stations in BSS 1 and one G.711, one video, one FTP stations in BSS 2. At time $300 s$, two video and two FTP connections from BSS 1 are started. At time $600 s$, these two video and two FTP connections are stopped, while one video and one FTP connections from BSS 2 are also stopped. These discrete events generate traffic variations during first $600 s$. We further introduce channel variations in BSS 2 at time $600 \mathrm{~s}$ by simulating NLOS transmissions in practice.

In scenario 2, we simulate a hotspot with two $802.11 \mathrm{~g}$ access points, operating with maximum data rate of $54 \mathrm{Mbps}$ and consider the case of mixed $802.11 \mathrm{~b} / \mathrm{g}$ stations as shown in Fig. 2 (b). We simulate channel variations by introducing NLOS transmission in an alternate fashion across both BSSs over a duration of $400 \mathrm{~s}$ each, starting from $100 \mathrm{~s}$ in BSS 1 . No traffic variations are simulated and therefore a similar load across both BSSs is used. Shadow fading is included for the entire simulation duration for both scenarios and the multimedia traffic sources are summarized in Table. I.

We further assume no hidden terminals and exclude RTSCTS mechanism from our simulation. However, CTS-to-self protection mechanism is used by $802.11 \mathrm{~g}$ stations. We also incorporate MAC service data unit (MSDU) lifetime limit mechanism to discard MSDUs from the transmitter queue if they exceed the MSDU lifetime before successful transmission. The MSDU lifetime for voice, video and data packets are chosen as $50 \mathrm{~ms}, 150 \mathrm{~ms}$ and $1 \mathrm{~s}$ respectively. All stations are roaming capable to support handover events.

The performance of our load optimization framework is investigated from three critical aspects. First, we quantify QoS performance as a function of two QoS metrics. Each QoS element is the ratio of the required QoS metric threshold and the measured QoS value. QoS satisfaction factor (QSF) is defined as the minimum between the two QoS elements,

$$
Q S F_{i}=\min _{i \in C}\left[\frac{P D_{t}^{i}}{P D_{m}^{i}}, \frac{P L R_{t}^{i}}{P L R_{m}^{i}}\right],
$$

where $P D_{t}^{i}$ is packet delay threshold, $P D_{m}^{i}$ is measured packet delay, $P L R_{t}^{i}$ is packet loss rate threshold, $P L R_{m}^{i}$ is measured packet loss rate of $i$ th service class and $Q S F_{i}<1$ when service QoS cannot be met.

Second, we quantify the effect of load optimization on achievable throughput of stations using the throughput fairness index (TFI),

$$
T F I_{i}=\frac{\left|\bar{S}_{m}^{i}-S_{t}^{i}\right|}{S_{t}^{i}}
$$

where $\bar{S}_{m}^{i}$ is measured average throughput and $S_{t}^{i}$ is target throughput for $i$ th service class. The throughput fairness index $0 \leq T F I \leq 1$ reflects the amount of deviation from target throughput and serves as a normalized measure of throughput fairness in the network. It has a value of 0 when throughput is fair and a value of 1 when throughput is tremendously unfair.

Last, we adopt Jain's fairness index to quantify the effect of load optimization on QoS metrics in each access point. Suppose $x_{i}$ is the PD or PLR of access point $i$, then the QoS balance index (QBI) can be defined as,

$$
Q B I(x)=\left(\sum_{i} x_{i}\right)^{2} / n\left(\sum_{i} x_{i}\right),
$$

where $n$ is the number of access points over which the load will be redistributed. The QoS balance index $0 \leq Q B I \leq 1$ is a continuous function which is independent of scale. It has a value of 1 when all access points have the exactly the same PD or PLR and a value of $1 / n$ when access points are extremely unbalanced, which is 0 in the limit as $n \rightarrow \infty$.

\section{Simulation Results}

The simulation results presented include QoS performance of our QoS-inspired load optimization (QLO) framework evaluated in terms of QSF, TFI and QBI. We then compare it with load balancing mechanism (LBM), representative of those implemented in [10] and [11], which does not consider wireless channel variations.

\section{A. Scenario 1}

According to the definitions in Eqs. 5-7, QSF should be greater than 1, TFI should be close to 0 and QBI should be close to 1, ideally to offer service QoS guarantee, throughput fairness and QoS fairness respectively. First, we study the effectiveness of QLO to support QoS demanding multimedia 
services in terms of QSF for stations. For this purpose, packet delay threshold for voice and video is $50 \mathrm{~ms}$, data is $300 \mathrm{~ms}$ and packet loss rate threshold for all service classes is $2 \%$. From Fig. 3 (a), the average QSFs for LBM and QLO are 37.3 and 40.6 respectively for the first $600 s$ where only traffic variations are simulated. However, QSF of 31.6 with LBM improves to 48.8 with QLO for the last $300 s$ when NLOS transmissions are introduced in BSS 2. Clearly, QLO outperforms LBM under such dissimilar channel conditions and provides comparable QoS support under similar channel conditions with traffic variations. Second, we examine the performance of QLO on throughput of stations using the TFI. From Fig. 3 (b), QLO exhibit throughput fairness with an average TFI of 0.08 when subjected to traffic variations during the first $600 \mathrm{~s}$ and dissimilar channel conditions during the last $300 \mathrm{~s}$. In contrast, LBM has an average TFI of 0.23 for the entire simulation. We note that under such disparate network conditions, LBM has a high TFI of 0.5 as some stations suffer huge deviations from their target throughput and hence fails to provide throughput fairness.

Last, we investigate the effect of QLO on packet delay and packet loss rate which are critical QoS metrics for supporting multimedia services, in particular, real-time traffic in both access points using the QBI. We analyze the results of Fig. 4 (a) in three segments which correspond to the simulated scenario. The simulation starts with an unbalanced load of fifteen stations in BSS 1 and three stations in BSS 2 during the first $300 s$. Over this period, there are eight handovers triggered by LBM in effort to balance the load between both access points but there are only two handovers triggered by QLO. The QBIs of packet delay for LBM and QLO are 0.71 and 0.85 respectively and the QBIs of packet loss rate for both LBM and QLO are similar with a value of 0.9. QLO achieves better performance as it handovers two voice stations, nominated as the 'best' station by access point 1 , which are the most aggressive source in this simulation. Although LBM uses the same algorithm to nominate the 'best' station, it tries to balance the load in a proactive manner. On the other hand, QLO takes the reactive approach such that handover is triggered only if packet loss rate of source access point exceeds $1 \%$ and when a better quality target access point exists. As a result, no handover is triggered by QLO during the next $300 s$ even when four additional stations are started in BSS 1 , whereas two additional handovers are triggered by LBM. It is for this reason that the QBI of packet delay for QLO drops to 0.68 while LBM maintains at relatively same level of 0.79. However, we emphasize that the service QoS are not compromised which is evident from Fig. 3 (a). The QBIs of packet loss rate for both LBM and QLO during the second $300 s$ are again similar with a value of 0.92 . We remark that is a direct consequence of our design principle of avoiding unnecessary handovers when service QoS can be supported.

In the last $300 s$, we introduce NLOS transmission in BSS 2 which essentially creates a disparate network conditions between both BSSs. Under such conditions, we notice from Fig. 4 (a) that QLO is still able to maintain high QBIs of both packet delay and packet loss rate with a value of 0.95 while QBIs of both packet delay and packet loss rate with LBM drops to 0.5 . This signifies that packet delay and packet loss rate between both access points are extremely unbalanced for the case with LBM. Hence, load balancing purely based on a single load metric such as channel utilization will fail under diverse channel conditions. As a final note, we highlight that there are a total of twelve handovers triggered by LBM but only two handovers with QLO. This translates to a significant $80 \%$ reduction in handovers while maintaining a high user QoS satisfaction factor and a QoS-balanced system.

\section{B. Scenario 2}

Here, we investigate the impact of channel variations on LBM and QLO. From Fig. 3 (a), it shows that average QSF of 40.8 for LBM can be improved to 71.9 with QLO. The key reason for this improvement is due to the capability of QLO in detecting station operating under bad channel conditions where the 'worst' station is identified as the one with the highest missed acknowledgments. From Fig. 3 (b), QLO preserves throughput fairness with a lower TFI of 0.15 as opposed to LBM of 0.38. We note that the two peaks in Fig. 3 (b) and two troughs in Fig. 4 (b) of QLO correspond to the adaptation time which detects and subsequently handovers the 'worst' station to a better quality access point. Accordingly, nine handovers are triggered with QLO during these adaptation period whereas no handover is triggered with LBM as shown in Fig. 4 (b). This reiterates the major pitfall of LBM which considers channel utilization as a single load metric, hence unable to respond to channel variations. Consequently, the QBIs of packet delay and packet loss rate in Fig. 4 (b) for QLO are 0.89 and 0.87 which outperform LBM's of 0.57 and 0.56 respectively.

Clearly, QLO is highly resilient to dynamic network conditions which may arise due to traffic and channel variations. In practice, many link adaptation algorithms are implemented by vendors to combat varying channel conditions. However, this gives rise to rate anomaly problem which has a serious implication on the perceived network capacity (cf. $§ I$ ). Specifically, rate anomaly in DCF would manifest as capacity outages if the arriving traffic is higher than the degraded system capacity caused by link adaptations. We argue that QLO can effectively minimize this capacity outage time by maintaining a QoSbalanced system where the 'worst' station can be detected and transferred to a better quality access point opportunistically. As soon as the 'worst' station is removed, it is expected that link adaptation procedure will act to increase the transmission rate, thus recovering the overall system capacity.

\section{CONClusion AND Future Work}

We present a QoS-inspired load optimization (QLO) framework which gives a unifying treatment in QoS provisioning for multimedia traffic over the widely deployed DCF-based WLANs. We show that load balancing with a single load metric such as channel utilization will fail under diverse channel conditions prevalent in hotspot deployments and indoor propagation environments. By introducing quadruple load metric, 

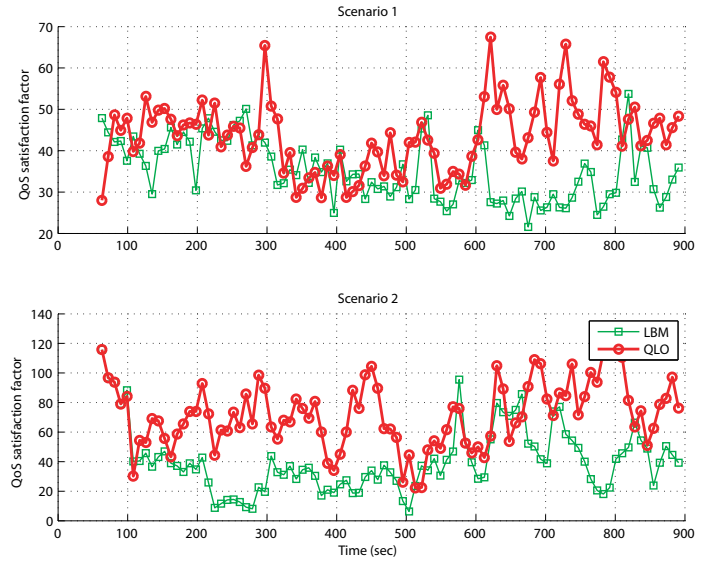

(a) QoS satisfaction factor.
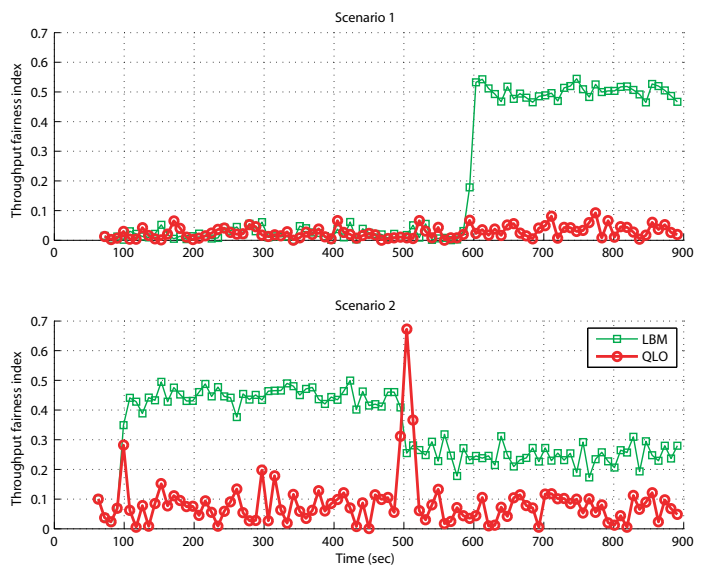

(b) Throughput fairness.

Fig. 3. QoS satisfaction factor and throughput fairness index.

we demonstrate that QLO provides adaptation to dynamic network conditions, which encompasses traffic and channel variations, in a self-adjusting manner to maintain a QoSbalanced system. We also show that a QoS-balanced system has intrinsic properties of providing service QoS guarantee while maximizing the overall system capacity. Hence, we advocate using QoS balance as load criterion in multiple access points based WLANs where network conditions are highly unpredictable. Finally, we argue that rate anomaly arising from link adaptation used to combat varying channel conditions can also be mitigated by maintaining a QoS-balanced system.

For future work, we would integrate link adaptation with our QLO framework to devise a harmonized solution for multirate WLAN. We are also working toward extension of QLO framework to scenarios with more than two access points and heterogeneous wireless networks.

\section{REFERENCES}

[1] H. Zhai, J. Wang, and Y. Fang. Providing statistical QoS guarantee for voice over IP in the IEEE 802.11 wireless LANs. IEEE Wireless Communications, 13(1):36-43, February 2006.

[2] E. H. Ong and J. Y. Khan. An integrated load balancing scheme for future wireless networks. In International Symposium on Wireless and Pervasive Computing, Melbourne, Australia, February 2009. to appear.

[3] J. Yu, S. Choi, and J. Lee. Enhancement of VolP over IEEE 802.11 WLAN via dual queue strategy. In Communications, 2004 IEEE International Conference on, volume 6, pages 3706-3711, June 2004.
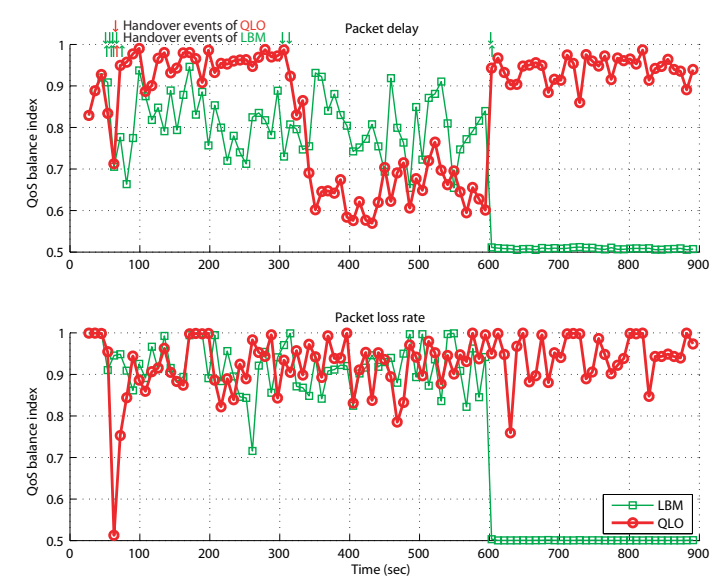

(a) Scenario 1.
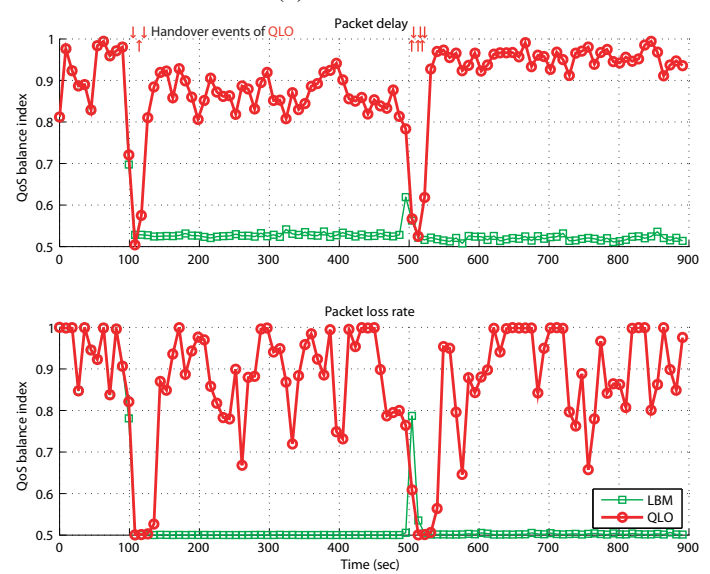

(b) Scenario 2 .

Fig. 4. QoS balance index of packet delay and packet loss rate.

[4] E. H. Ong and J. Y. Khan. Dynamic access network selection with QoS parameters estimation: A step closer to ABC. In Vehicular Technology Conference, 2008. VTC Spring 2008. IEEE, pages 2671-2676, Marina Bay, Singapore, May 2008.

[5] M. Heusse, F. Rousseau, G. Berger-Sabbatel, and A. Duda. Performance anomaly of $802.11 \mathrm{~b}$. In INFOCOM 2003. Twenty-Second Annual Joint Conference of the IEEE Computer and Communications Societies. IEEE, volume 2, pages 836-843, March/April 2003.

[6] G. Tan and J. Guttag. Time-based fairness improves performance in multi-rate wlans. In ATEC '04: Proceedings of the annual conference on USENIX Annual Technical Conference, pages 23-23, Berkeley, CA, USA, 2004. USENIX Association.

[7] G. Bianchi and I. Tinnirello. Improving load balancing mechanisms in wireless packet networks. In Communications, 2002. ICC 2002. IEEE International Conference on, volume 2, pages 891-895, 2002.

[8] E. H. Ong and J. Y. Khan. QoS provisioning for VoIP over wireless local area networks. In 11th IEEE International Conference on Communication Systems 2008, pages 906-911, Guangzhou, P.R. China, November 2008.

[9] S. Garg and M. Kappes. Admission control for VoIP traffic in IEEE 802.11 networks. In Global Telecommunications Conference, 2003. GLOBECOM '03. IEEE, volume 6, pages 3514-3518, December 2003.

[10] R. Daher and D. Tavangarian. Qos-oriented load balancing for WLANs. In Operator-Assisted (Wireless Mesh) Community Networks, 2006 1st Workshop on, pages 1-12, September 2006.

[11] H. Velayos, V. Aleo, and G. Karlsson. Load balancing in overlapping wireless LAN cells. In Communications, 2004 IEEE International Conference on, volume 7, pages 3833-3836, June 2004.

[12] T. Rappaport. Wireless Communications: Principles and Practice. Prentice Hall PTR, Upper Saddle River, NJ, USA, 2001. 